This item was submitted to Loughborough's Research Repository by the author.

Items in Figshare are protected by copyright, with all rights reserved, unless otherwise indicated.

\title{
A stable model predictive control algorithm without terminal weighting
}

PLEASE CITE THE PUBLISHED VERSION

PUBLISHER

(c) Sage

LICENCE

CC BY-NC-ND 4.0

\section{REPOSITORY RECORD}

Chen, Wen-Hua, and Xiao-Bing Hu. 2008. "A Stable Model Predictive Control Algorithm Without Terminal Weighting”. figshare. https://hdl.handle.net/2134/3772. 
This item was submitted to Loughborough's Institutional Repository (https://dspace.lboro.ac.uk/) by the author and is made available under the following Creative Commons Licence conditions.

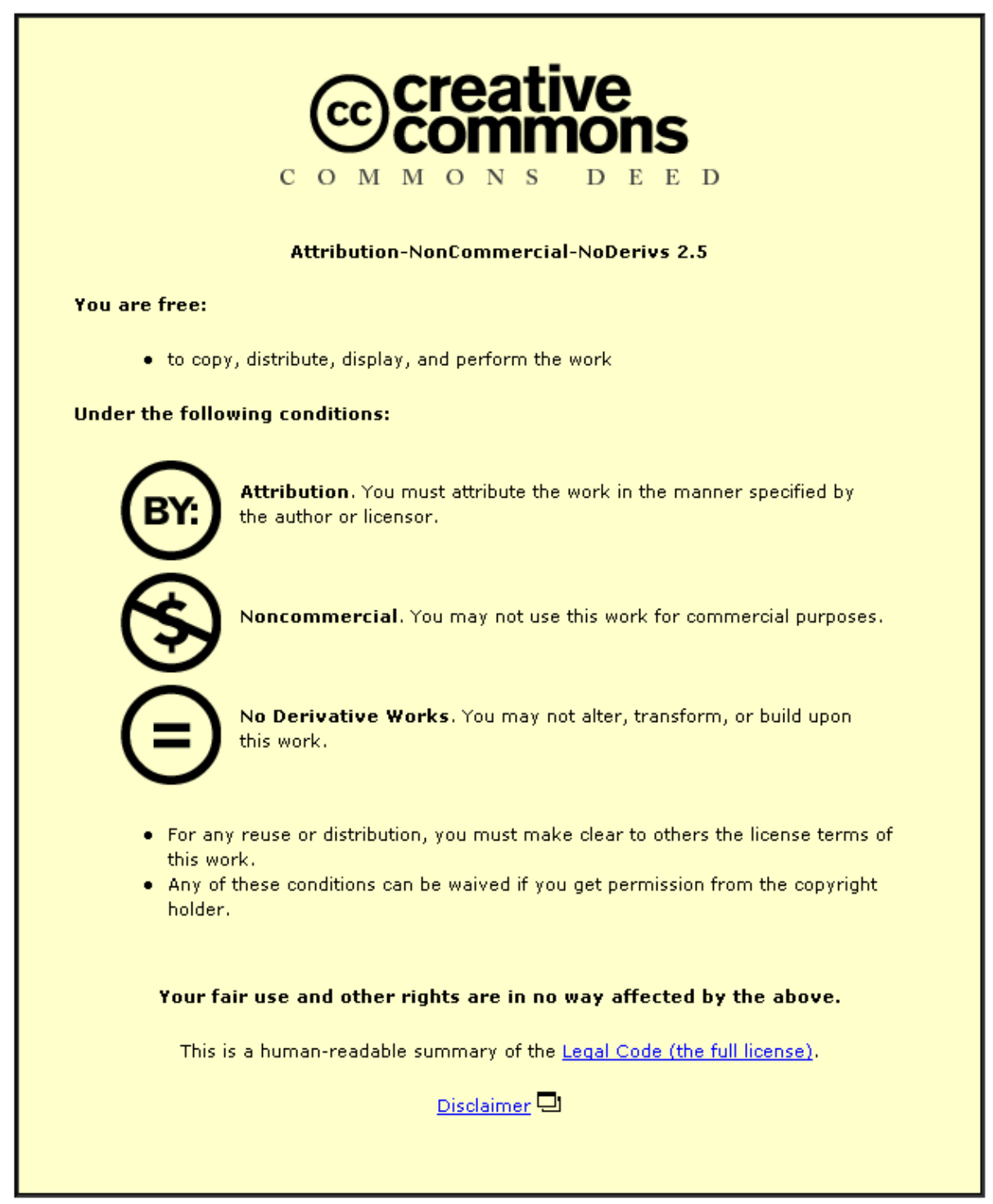

For the full text of this licence, please go to: http://creativecommons.org/licenses/by-nc-nd/2.5/ 


\title{
A Stable Model Predictive Control Algorithm without Terminal Weighting
}

\author{
Wen-Hua Chen and Xiao-Bing Hu \\ Department of Aeronautical and Automotive Engineering \\ Loughborough University \\ Loughborough \\ Leicestershire LE11 3TU \\ UK \\ Tel.: +44 1509227230 \\ Fax: +44 1509227275 \\ Email: W.Chen@1boro.ac.uk,X.Hu@1boro.ac.uk
}




\begin{abstract}
:
The introduction of terminal penalty in the performance index and the usage of the concept of terminal regions now become common practice in Model Predictive Control (MPC) for guaranteeing its stability. However, it is quite difficult and conservative to propagate the influence of disturbances and uncertainties from an initial state to the terminal state, in particular, when the predictive horizon is long. This paper presents a new stable MPC algorithm where the additional weighting on the first state rather than on the terminal state in the horizon is imposed. Furthermore, a new tuning knob is introduced in the performance index, which can be used to trade off between disturbance attenuation/robustness and stability. It is shown that in the absence of disturbances and uncertainties, the new MPC algorithm achieves the similar performance as current terminal weighting based MPC algorithms. However, it exhibits much better disturbance attenuation ability and robustness against uncertainties. The proposed method is favorably compared with terminal weighting based MPC algorithms by a numerical example.
\end{abstract}

Keywords: Model Predictive Control, Robustness, Disturbance Attenuation, Linear Matrix Inequalities, Stability.

\title{
1. Introduction
}

Model Predictive Control (MPC) has been widely adopted in industry (Garcia et al, 1989, Clarke, 1994, Mayne et al, 2000 and Maciejowski 2002). Stability has been one of the main problems in MPC since early MPC was criticized for its loss of stability, see Bitmead et al (1990). After two decades' study, stability of MPC is now reaching its premature stage and many methods have been presented. As discussed in Mayne et al (2000), the core idea behind most of these methods is to add a terminal weighting term in the performance index and impose constraints on the state in the end of the horizon, i.e., the terminal state, being within a region, referred to as a terminal region, to address stability and feasibility. The MPC algorithms developed based on this concept are referred to as terminal weighting based MPC (TW-MPC) in this paper. This idea has also 
been extended from linear systems to nonlinear systems (see Mayne and Michalska, 1990, Rawlings and Muske, 1993 and Chen et al, 2000). How to choose the terminal weighting term and determine the terminal region thus stability region have been discussed in Lee et al (1998) for linear systems and in Chen and Allgower (1998) and Chen et al (2001) for nonlinear systems. The importance of calculation of the stability region is significant since it provides a region where the MPC algorithm can work.

Most of the engineering systems are subject to disturbances and uncertainties. When the TW-MPC algorithms are applied, it is quite difficult and conservative to propagate the influence of disturbances and uncertainties from an initial state to the terminal state, which should be within a terminal region determined by stability or feasibility requirements. This results in the difficulties and conservativeness in analysis and design of MPC for systems under disturbances or with uncertainties.

The first purpose of this paper is to propose a new MPC scheme to overcome the above mentioned shortcomings of the TW-MPC algorithms. Unlike the TW-MPC algorithms where the stability is achieved by inspecting the terminal state, the algorithm proposed in this paper guarantees the stability by inspecting the first state in the predictive horizon. In other words, an extra weighting on the first state in the horizon is added in the performance index, which leads to that the first state holds certain properties, and then the stability is established based on this. This new MPC algorithm is referred to as first state weighting based MPC algorithm (FW-MPC). This is motivated by the observation that only the first control action in the control sequence yielded by online optimization is implemented in MPC, and as long as the first state holds certain properties, it is possible to establish stability for the whole MPC scheme.

The idea to establish stability based on the first state in the horizon has appeared in the stability enforced MPC algorithms, where stability is achieved by enforcing the state to contract to the origin in each step, see e.g. Sznaier and Damborg (1990), Polak and Yang (1993), Bemporad (1998) and Kothare and Morari (2000). In order to do so, an extra constraint (usually, a Lyapunov function alike) is added in the online optimization problem. To this end, perhaps the earliest algorithm is due to Sznaier and Damborg (1990), who employ an auxiliary Lyapunov function whose level set $\{x \mid v(x) \leq 1\}$ is the state constraint set. An extra stability constraint $v(f(x, u))<v(x)$ is added to the optimal 
control problem. Although the stability enforced MPC algorithms have their own advantages, in contrast to the widely used TW-MPC, it does not have methods to calculate the stability/feasibility region offline. Therefore, the region where the MPC algorithm is applicable/feasible is not known priori and no feasible initial control sequence is available. The latter is quite important for guaranteeing stability and performance of MPC when on-line optimization cannot be completed within a given sampling interval (Mayne, et al, 2000). The FW-MPC tries to retain the features of TWMPC and the performance index is modified to enforce the state to contract to the origin in each step.

The robust MPC algorithm proposed in Kothare et al (1996) also combines some features of both TW-MPC and stability-enforced MPC, but in a different way as FWMPC does. An infinite horizon performance index is adopted by this MPC algorithm and the purpose of online optimization is to minimize the worst performance under uncertainties. Like other stability-enforced MPC algorithms, an additional constraint is imposed on the online optimization for the stability purpose, but the additional constraint is similar to those stabilizing conditions for TW-MPC algorithms. In view of the large amount of computational burden imposed by this algorithm, Kouvaritakis et al (2000) and Kouvaritakis et al (2002) refined this algorithm and developed much more computationally efficient MPC algorithms by shifting most of the computational burden from online to offline.

When a system is subject to unknown disturbances, in general, asymptotic stability cannot be achieved by an MPC algorithm any more. In this case, not only the stability/feasibility region but also disturbance attenuation ability is concerned. The second purpose of this paper is to understand the influence of the design parameters on the disturbance attenuation ability and to provide a way to trade off between the stability region, performance and disturbance attenuation ability. Two indices are used to describe the disturbance attenuation ability of MPC: the allowable disturbances under which the MPC can maintain its stability and the level the MPC can attenuate the disturbance to. The latter is defined by a region where once state enters, it remains within it.

This paper is organized as follows: In Section 2 the constrained MPC problem for linear systems is formulated and then the FW-MPC algorithm is proposed. The properties 
of the FW-MPC are analyzed in Section 3 to 5, including stability, disturbance attenuation and robustness against uncertainties. The influence of the design parameters in the FW-MPC is discussed in Section 6. Simulation results are reported in Section 7 and the paper ends with conclusions in Section 8.

\section{FW-MPC algorithm}

Consider a linear discrete-time system

$$
\left\{\begin{aligned}
x(k+1) & =A x(k)+B u(k) \\
x(0) & =x_{0}
\end{aligned}\right.
$$

with control constraints

$$
\left|u_{i}\right| \leq \bar{u}_{i}, \quad i=1, \cdots, m
$$

where $k$ is the discrete time index, $x \in R^{n}$ represents the system state, $u \in R^{m}$ is the input vector, and $\bar{u}_{i}, i=1, \cdots, m$ is the input constraints.

The original MPC performance index is given by

$$
J(k)=\sum_{i=0}^{N-1}\left(x(k+i \mid k)^{T} Q x(k+i \mid k)+u(k+i \mid k)^{T} R u(k+i \mid k)\right),
$$

where $Q>0$ (or $Q \geq 0$ and $\left[A, Q^{1 / 2}\right]$ is detectable) and $R>0$ are state and control weighting matrices respectively, and $N$ is the length of the predictive horizon.

Since MPC algorithm based on the performance index (3) may lose its stability, in TW-MPC algorithms, the stability is achieved by adding the weighting on the terminal state, $x(k+N \mid k)$, in the performance index $J(k)$, that is,

$$
\begin{aligned}
J_{1}(k)=x(k & +N \mid k)^{T} P x(k+N \mid k)+ \\
& +\sum_{i=0}^{N-1}\left(x(k+i \mid k)^{T} Q x(k+i \mid k)+u(k+i \mid k)^{T} R u(k+i \mid k)\right),
\end{aligned}
$$

where $P>0$ is the terminal weighting matrix.

Then the stability of the TW-MPC can be established if the terminal weighting term and the terminal state satisfy certain conditions. However, when the system (1) is subject to disturbances or (and) uncertainties, it is quite difficult and conservative to establish the stability by inspecting the behaviour of the terminal state $x(k+N \mid k)$ since the 
disturbances and the uncertainties in the receding horizon, $k, \ldots, k+N-1$ have the influence on the terminal state in a quite complicated way.

A new performance index, which emphasizes the first predicted state rather than the last one in the horizon, is proposed in this paper

$$
\begin{aligned}
J_{2}(k)=x(k+1 \mid k)^{T} M x(k+1 \mid k)+ & \\
& +\sum_{i=0}^{N-1} x(k+i \mid k)^{T} Q x(k+i \mid k)+u(k+i \mid k)^{T} R u(k+i \mid k),
\end{aligned}
$$

where $M$ is the weighting matrix for the first predicted state.

Similar to the current TW-MPC algorithms, at each step, the performance index (5) is minimized on-line, that is,

$$
\min _{u(k \mid k), \cdots, u(k+N-1 \mid k)} J_{2}(k)
$$

subject to (1) and (2) by using an optimization solver and the optimal control sequence $u^{*}(k \mid k), \cdots, u^{*}(k+N-1 \mid k)$ is yielded. Then the MPC law is determined by

$$
u(k)=u^{*}(k \mid k) .
$$

\section{Exponential Stability of FW-MPC}

Let the control effort in each time instant be given by

$$
u(k+i \mid k)=K(k+i) x(k), i=0, \ldots, N-1,
$$

where $K(k+i)$ is the control gain at time instant $k+i$.

Then the predicted state at time instant $k+i$ driven by the control sequence $u(k \mid k), \ldots, u(k+N-1 \mid k)$ from the state $x(k)$ can be expressed by

$$
x(k+i \mid k)=\left(A^{i}+\left[\begin{array}{lll}
A^{i-1} B & \cdots & A^{0} B
\end{array}\right] \cdot K_{i}(k)\right) \cdot x(k), \quad i=1, \ldots, N
$$

where

$$
K_{i}(k)=\left[\begin{array}{lll}
K(k)^{T} & \cdots & K(k+i-1)^{T}
\end{array}\right]^{T}, i=1, \ldots, N .
$$

Substituting Eq. (9) into the performance index (5) gives

$$
\begin{aligned}
J_{2}(k)= & x(k)^{T}\left((A+B K(k))^{T} M(A+B K(k))+\right. \\
& \left.+\left(\widetilde{\Phi}_{N}+\widetilde{\Gamma}_{N} K_{N}(k)\right)^{T} Q_{N}\left(\widetilde{\Phi}_{N}+\widetilde{\Gamma}_{N} K_{N}(k)\right)+K_{N}(k)^{T} R_{N} K_{N}(k)\right) x(k) \\
= & x(k)^{T}\left((A+B K(k))^{T}(M+Q)(A+B K(k))+\right.
\end{aligned}
$$




$$
\left.+\left(\Phi_{N}+\Gamma_{N} K_{N}(k)\right)^{T} Q_{N}\left(\Phi_{N}+\Gamma_{N} K_{N}(k)\right)+K_{N}(k)^{T} R_{N} K_{N}(k)\right) x(k)
$$

where

$$
\begin{aligned}
& \widetilde{\Gamma}_{N}=\left[\begin{array}{ccccc}
0 & 0 & 0 & \cdots & 0 \\
B & 0 & 0 & \cdots & 0 \\
A B & B & 0 & \cdots & 0 \\
\vdots & \vdots & \vdots & \ddots & \vdots \\
A^{N-2} B & A^{N-3} B & \cdots & B & 0
\end{array}\right], \Gamma_{N}=\left[\begin{array}{ccccc}
0 & 0 & 0 & \cdots & 0 \\
0 & 0 & 0 & \cdots & 0 \\
A B & B & 0 & \cdots & 0 \\
\vdots & \vdots & \vdots & \ddots & \vdots \\
A^{N-2} B & A^{N-3} B & \cdots & B & 0
\end{array}\right], \\
& \widetilde{\Phi}_{N}=\left[\begin{array}{llllll}
I & A^{T} & \left(A^{2}\right)^{T} & \cdots & \left(A^{N-1}\right)^{T}
\end{array}\right]^{T}, \Phi_{N}=\left[\begin{array}{lllll}
I & 0 & \left(A^{2}\right)^{T} & \cdots & \left(A^{N-1}\right)^{T}
\end{array}\right]^{T}
\end{aligned}
$$

and

$$
Q_{N}=\operatorname{diag}\{\underbrace{\{Q, \ldots, Q\}}_{N}, \quad R_{N}=\operatorname{diag} \underbrace{\{R, \ldots, R\}}_{N} .
$$

Definition 1: Stability region $v$ refers to a set of all initial state $x_{0}$ from which the system state under the MPC stemming from the optimization problem (6) subject to constraints (2) approaches to the origin, i.e., $x(k) \rightarrow 0$ as $k \rightarrow 0$ in the absence of disturbance.

Theorem 1: Suppose that there exist matrices $S>0, \bar{S}$ and scalars $\mu>0, e>1$ such that

$$
\left[\begin{array}{cccc}
-S & \left(A S+B S_{0}\right)^{T} & \left(\Phi_{N}^{Q} S+\Gamma_{N}^{Q} \bar{S}\right)^{T} & \bar{S}^{T} \\
A S+B S_{0} & -S / e^{2} & 0 & 0 \\
\Phi_{N}^{Q} S+\Gamma_{N}^{Q} \bar{S} & 0 & -\mu I & 0 \\
\bar{S} & 0 & 0 & -\mu\left(R_{N}\right)^{-1}
\end{array}\right] \leq 0
$$

and

$$
\left[\begin{array}{cc}
Y & S_{i} \\
\left(S_{i}\right)^{T} & S
\end{array}\right] \geq 0, \quad Y_{j j} \leq \bar{u}_{j}^{2}, \quad i=0, \ldots, N-1 ; j=1, \ldots, m
$$

hold where

$$
\begin{gathered}
\bar{S}=\left[\begin{array}{lll}
\left(S_{0}\right)^{T} & \cdots & \left(S_{N-1}\right)^{T}
\end{array}\right]^{T}, \\
\Phi_{N}^{Q}=\left(Q_{N}\right)^{1 / 2} \Phi_{N}, \Gamma_{N}^{Q}=\left(Q_{N}\right)^{1 / 2} \Gamma_{N},
\end{gathered}
$$

$\Phi_{N}, \Gamma_{N}, Q_{N}$ and $R_{N}$ are given in (12), (13) and (14). Then the FW-MPC with the predictive performance index (5) exponentially stabilizes the constrained linear system (1) for all initial states within the set 


$$
v=\left\{x \in R^{n}: x^{T} Z x \leq \mu\right\}
$$

if the extra weighting matrix for the first predicted state is chosen as

$$
M=e Z e-Q
$$

where $Z=S^{-1} \mu$.

Proof: Suppose that there exist $\mu>0, e>1,0<Z \in R^{n \times n}, K(k), \ldots, K(k+N-1)$ such that

$$
\begin{aligned}
Z \geq( & A+B K(k))^{T} e Z e(A+B K(k))+ \\
& +\left(\Phi_{N}+\Gamma_{N} K_{N}(k)\right)^{T} Q_{N}\left(\Phi_{N}+\Gamma_{N} K_{N}(k)\right)+K_{N}(k)^{T} R_{N} K_{N}(k)
\end{aligned}
$$

and

$$
\left[\begin{array}{cc}
Y & K(k+i) \\
K(k+i)^{T} & Z / \mu
\end{array}\right] \geq 0, \quad Y_{j j} \leq \bar{u}_{j}^{2}, \quad j=1, \ldots, m ; i=0, \ldots, N-1 .
$$

Condition (22) implies that the state feedback control sequence, $\widetilde{u}(k+i \mid k)=K(k+i) x(k), i=0, \ldots, N-1$, satisfies the control constraints (2) if $x(k) \in v$, i.e., $x(k)^{T} Z x(k) \leq \mu$ ( Kothare et al, 1996).

Let $\widetilde{x}(k+i \mid k)$ denote the predicted state under $\widetilde{u}(k+i \mid k)$ and $\widetilde{J}_{2}(k)$ denote the corresponding performance index. Similarly, $x^{*}(k+i \mid k)$ and $J_{2}^{*}(k)$ denote the state under the optimal control sequence yielded by solving the on-line optimization problem and the corresponding performance index, respectively. It follows from the principle of optimality, Eqs. (11), (20) and condition (21) that

$$
\begin{aligned}
x^{*}(k+1 \mid k)^{T} \operatorname{eZex}^{*}(k+1 \mid k) \leq J_{2}^{*}(k) \leq \widetilde{J}_{2}(k) \\
=\widetilde{x}(k+1 \mid k)^{T} M \widetilde{x}(k+1 \mid k)+ \\
\quad+\sum_{i=0}^{N-1} \widetilde{x}(k+i \mid k)^{T} Q \widetilde{x}(k+i \mid k)+\widetilde{u}(k+i \mid k)^{T} R \widetilde{u}(k+i \mid k) \\
=x(k)^{T}\left[(A+B K(k))^{T}(M+Q)(A+B K(k))+\right. \\
\left.\quad+\left(\Phi_{N}+\Gamma_{N} K_{N}(k)\right)^{T} Q_{N}\left(\Phi_{N}+\Gamma_{N} K_{N}(k)\right)+K_{N}(k)^{T} R_{N} K_{N}(k)\right] x(k) \\
=x(k)^{T}\left[(A+B K(k))^{T} e Z e(A+B K(k))+\right. \\
\left.\quad+\left(\Phi_{N}+\Gamma_{N} K_{N}(k)\right)^{T} Q_{N}\left(\Phi_{N}+\Gamma_{N} K_{N}(k)\right)+K_{N}(k)^{T} R_{N} K_{N}(k)\right] x(k) \\
\leq x(k)^{T} Z x(k) .
\end{aligned}
$$


In MPC the first control action in the optimal sequence is executed. If there are no disturbances and no system uncertainties, one has

$$
x(k+1)=x^{*}(k+1 \mid k) .
$$

Hence (23) implies that

$$
x(k+1)^{T} e \operatorname{Zex}(k+1) \leq x(k)^{T} Z x(k) .
$$

It follows from $e>1$ that $x(k+1)$ contracts to the origin.

Conditions (21) and (22) can be transferred into conditions (15) and (16) by using the transform

$$
Z=S^{-1} \mu, K_{N}(k)=\bar{S} S^{-1} \text {, and } K(k+i)=S_{i} S^{-1} .
$$

Since conditions (15) and (16) imply that condition (24) holds for any $k=0,1, \ldots$, it can be shown that for any initial state in the set $v$, the trajectory of $x(\cdot)$ converges to the origin exponentially. This completes the proof.

Remark 1: At each time instant, after the state is measured, the initial control sequence for the online optimization is chosen as $\widetilde{u}(k+i \mid k)=K(k+i) x(k)$, $i=0, \ldots, N-1$, and the associated performance index is given by $\widetilde{J}_{2}(k)$. Even if the computational time runs out before the optimization is completed, the first predicted state $\tilde{x}^{*}(k+1 \mid k)$ under the latest control sequence yielded by the optimizer and the corresponding performance index $\widetilde{J}_{2}^{*}(k)$ still satisfy

$$
\widetilde{x}^{*}(k+1 \mid k)^{T} e Z e \widetilde{x}^{*}(k+1 \mid k) \leq \widetilde{J}_{2}^{*}(k) \leq \widetilde{J}_{2}(k) \leq x(k)^{T} Z x(k),
$$

which implies that the FW-MPC is always feasible and exponentially stable.

Remark 2: According to Theorem 1, the system under the FW-MPC is exponentially stable when all states are available. For the output feedback case, by integrating a state estimator with exponential stability with the FW-MPC algorithm, as demonstrated in Scokaert et al (1997), asymptotic stability can be achieved.

Remark 3: In Theorem 1 the weighting matrix $M$ on the first predicted state is determined by (20) and $e>1$ is an extra design parameter introduced in this paper. As it will be seen, the parameter $e$ provides a tuning knob to trade off between the stability region and disturbance attenuation/robustness of the FW-MPC. 


\section{Disturbance attenuation}

This section investigates the disturbance attenuation ability of the MPC algorithm proposed in Section 3. To this end, suppose that the constrained system (1) is subject to a bounded disturbance, that is,

$$
\left\{\begin{array}{l}
x(k+1)=A x(k)+B u(k)+C d(k) \\
x(0)=x_{0}, \quad\left|u_{i}\right| \leq \bar{u}_{i}, \quad i=1, \cdots, m
\end{array},\right.
$$

where the disturbance is bounded by

$$
d(k)_{i} \in\left\lfloor\underline{D_{i}}, \overline{D_{i}}\right\rfloor, \quad i=1, \ldots, l .
$$

It is obvious that it is impossible to achieve asymptotic stability for a MPC algorithm under disturbances. Furthermore, due to the constraints imposed on the system, different from unconstrained linear systems, the stability of the MPC may be destroyed by disturbances. Hence, the size of the stability region depends on the range of the disturbances.

To establish our results, the following definitions are necessary.

Definition 2 (Corless and Leitmann, 1981): System (26) under the FW-MPC and the bounded disturbance (27) is said to be uniformly ultimately bounded if there exists a neighborhood of the origin $x=0, \Theta$, such that the state trajectory enters it in finite time and remains within it thereafter. The set $\Theta$ is referred to as Uniform Ultimate Boundedness Region (UUBR). The set of all the initial state $x_{0}$ from which the system (26) under the FW-MPC and the bounded disturbances (27) is uniformly ultimately bounded is referred to as the stability region under the disturbances.

Definition 3: Maximum Allowable Disturbance Set (MADS) $\Phi$ is referred to as a set of allowable bounded disturbances under which for all the states starting from the stability region $v$, the system (26) under the FW-MPC remains stable.

Theorem 2: Consider the constrained system (26), (27) with the MPC performance index (5), where $M$ is determined by (20). Suppose that there exist matrices $S>0, \bar{S}$ and a scalar $\mu>0$ such that conditions (15) and (16) are satisfied. Then

(1). the MADS is 


$$
\Phi=\left\{d(k) \in R^{l} \mid\left(\frac{e}{e-1}\right)^{2} d(k)^{T} C^{T} Z C d(k) \leq \mu\right\}
$$

where $Z=S^{-1} \mu$;

(2). when the disturbance belongs to the set (28), for any initial state $x_{0} \in v$ as defined in (19), the FW-MPC can steer the state into the UUBR

$$
\Theta=\left\{x \in R^{n} \mid x^{T} Z x \leq\left(\frac{e}{e-1}\right)^{2} \hat{d}(k)^{T} C^{T} Z C \hat{d}(k)\right\},
$$

where $\hat{d}(k)$ is the disturbance which maximizes $d(k)^{T} C^{T} Z C d(k)$ subject to (27).

Proof: Similar to the proof of Theorem 1, it can be shown that in the absence of disturbances, the FW-MPC satisfying conditions (15) and (16) guarantees that

$$
x^{*}(k+1 \mid k)^{T} e Z e x^{*}(k+1 \mid k) \leq x(k)^{T} Z x(k)
$$

where $x^{*}(k+1 \mid k)$ is the first predicted state under the control $u^{*}(k \mid k)$.

Since $Z>0$, it can be decomposed as $Z=U^{T} U$ where $U$ is of full rank. Since $e>0$, using the transformation $\bar{x}(\cdot)=U x(\cdot)$, Eq. (30) becomes

$$
\left\|\bar{x}^{*}(k+1 \mid k)\right\|^{2} e^{2} \leq\|\bar{x}(k)\|^{2} \Rightarrow\left\|\bar{x}^{*}(k+1 \mid k)\right\| e \leq\|\bar{x}(k)\| .
$$

When a bounded disturbance is present, the actual state $x(k+1)$ is different from the first predicted state, $x^{*}(k+1 \mid k)$, i.e., $x(k+1)=x^{*}(k+1 \mid k)+C d(k)$, which implies

$$
\bar{x}(k+1)=\bar{x}^{*}(k+1 \mid k)+U C d(k) .
$$

Combining (31) with (32) yields

$$
\begin{aligned}
\|\bar{x}(k+1)\| e & =\left\|\bar{x}^{*}(k+1 \mid k)+U C d(k)\right\| e \leq\left\|\bar{x}^{*}(k+1 \mid k)\right\| e+\|U C d(k)\| e \\
& \leq\left\|\bar{x}^{*}(k+1 \mid k)\right\| e+\|U C \hat{d}(k)\| e \leq\|\bar{x}(k)\|+\|U C \hat{d}(k)\| e \\
& =\|\bar{x}(k)\| e+\|\bar{x}(k)\|(1-e)+\|U C \hat{d}(k)\| e .
\end{aligned}
$$

When $(1-e)\|\bar{x}(k)\|+e\|U C \hat{d}(k)\|<0$, one has that $\|\bar{x}(k+1)\|<\|\bar{x}(k)\|$ from (33). Since $e>1$, this implies that any state satisfying $\|\bar{x}(k)\|>\frac{e}{e-1}\|U C \hat{d}(k)\|$ contracts to the region 
$\Theta$ until $\|\bar{x}(k)\| \leq \frac{e}{e-1}\|U C \hat{d}(k)\|$. Therefore, any state outside of the region $\Theta$ in (29) will finally enter it.

Now we prove that once the state enters the region $\Theta$, it will remain in that region. Suppose that in time instant $k$, the state belongs to the region $\Theta$ and hence satisfies the condition $\|\bar{x}(k)\| \leq \frac{e}{e-1}\|U C \hat{d}(k)\|$. It follows from (33) that

$$
\begin{aligned}
\|\bar{x}(k+1)\| e & \leq \frac{e}{e-1}\|U C \hat{d}(k)\|+\|U C \hat{d}(k)\| e=\frac{e^{2}}{e-1}\|U C \hat{d}(k)\| \\
& \Rightarrow\|\bar{x}(k+1)\| \leq \frac{e}{e-1}\|U C \hat{d}(k)\| .
\end{aligned}
$$

Therefore the state remains in the region $\Theta$ once it enters.

When the state trajectory starts from any initial state within the stability region $v$, a sufficient condition for the system being stable under the disturbances is that the state in the next step still remains in the region $v$. From the above proof, it can be seen that if the disturbance satisfies

$$
\left(\frac{e}{e-1}\right)^{2} \hat{d}(k)^{T} C^{T} Z C \hat{d}(k) \leq \mu,
$$

then for any $x(k) \in v$, the state in the next step under the FW-MPC still remains in the stability region $v$. This is because

$$
x(k+1)^{T} Z x(k+1)=\|\bar{x}(k+1)\| \leq \frac{e}{e-1}\|U C \hat{d}(k)\|=\left(\frac{e}{e-1}\right)^{2} \hat{d}(k)^{T} C^{T} Z C \hat{d}(k) \leq \mu .
$$

Hence the MADS and UUBR are determined by (28) and (29) respectively.

Remark 4: Since $C^{T} Z C$ is semi-positive definite, the maximum of $d(k) C^{T} Z C d(k)$ achieves at a certain extreme point of $d(k)$.

\section{Robustness}

Robustness is another important property of control methods. This section addresses the robustness issue of the FW-MPC algorithm. It is supposed that there are uncertainties in the system matrix described by

$$
A=A_{o}+A_{u}
$$


where $A_{o}$ is a nominal system matrix and $A_{u}$ is the perturbation matrix which is within a given set

$$
A_{u} \in \Omega .
$$

In general, it is reasonable to assume that the set $\Omega$ is convex or can be covered by a convex hull of a set of extreme matrices $\left[\bar{A}_{u 1}, \ldots, \bar{A}_{u L}\right]$ (Kothare et al, 1996). Thus, any $A_{u} \in \Omega$ can be represented by a linear combination of these extreme matrices

$$
A_{u}=\sum_{i=1}^{L} \alpha_{i} \bar{A}_{u, i}, \sum_{i=1}^{L} \alpha_{i}=1, \alpha_{i} \geq 0 .
$$

Theorem 3: Suppose that there exist matrices $S>0, \bar{S}$ and a scalar $\mu>0$ such that conditions (15) and (16) hold for the nominal system and

$$
\left[\begin{array}{cc}
S & \left(\bar{A}_{u, i} S\right)^{T} \\
\bar{A}_{u, i} S & \left(\frac{e-1}{e}\right)^{2} S
\end{array}\right]>0, i=1, \ldots, L
$$

is satisfied. Then the FW-MPC with first predicted state weighting $M$, which is determined by (20), can asymptotically stabilize the uncertain system defined by (35) and (36) for all initial states in the region $v$ in (19).

Proof: Similar to the proof for Theorem 2, when conditions (15) and (16) hold for the nominal system, the first predicted state satisfies (30), and then (31) holds.

In the presence of system uncertainties, the actual state, which is different from the first predicted state based on the nominal system, is given by

$$
\begin{aligned}
x(k+1)= & \left(A_{o}+A_{u}\right) x(k)+B u(k)=x^{*}(k+1 \mid k)+A_{u} x(k) \\
& \Rightarrow \bar{x}(k+1)=\bar{x}^{*}(k+1 \mid k)+U A_{u} x(k),
\end{aligned}
$$

which implies

$$
\|\bar{x}(k+1)\| e \leq\left\|\bar{x}^{*}(k+1 \mid k)\right\| e+\left\|U A_{u} x(k)\right\| e \leq\|\bar{x}(k)\|+\left\|U A_{u} x(k)\right\| e .
$$

If $\frac{1-e}{e}\|\bar{x}(k)\|+\left\|U A_{u} x(k)\right\|<0$, then one can conclude that $\|\bar{x}(k+1)\|<\|\bar{x}(k)\|$, which means that the state trajectory converges. Therefore any state satisfying $\|\bar{x}(k)\|>\frac{e}{e-1}\left\|U A_{u} x(k)\right\|$ converges to the origin. This condition is equivalent to 


$$
x(k)^{T} Z x(k)>\left(\frac{e}{e-1}\right)^{2} x(k)^{T} A_{u}^{T} Z A_{u} x(k),
$$

which is met if

$$
Z-\left(\frac{e}{e-1}\right)^{2} A_{u}^{T} Z A_{u}>0 .
$$

Using the transform $Z=S^{-1} \mu$, it is shown that condition (40) is equivalent to

$$
\left[\begin{array}{cc}
S & \left(A_{u} S\right)^{T} \\
A_{u} S & \left(\frac{e-1}{e}\right)^{2} S
\end{array}\right]>0 .
$$

Since $A_{u} \in \Omega$ and can be represented by (37), one has that (41) holds if (38) holds. i

\section{Design parameters in the FW-MPC algorithms}

A large $e$ means a heavy penalty imposed on the first predicted state, which implies a high convergence rate of the FW-MPC algorithm in the absence of system uncertainties and disturbances, or, in general, a good disturbance attenuation ability/strong robustness in the presence of disturbances/uncertainties. Unfortunately, a large $e$ also leads to a small stability region. Hence $e$ should be chosen based on the trade off between the stability region and convergence rate/disturbance attenuation ability/robustness.

After a proper $e$ is chosen, the matrix $Z$ is determined by solving offline the optimization problem

$$
\min _{S>0, \bar{S}, \mu>0} \log \left(\operatorname{det}\left(S^{-1}\right)\right)
$$

subject to conditions (15) and (16). The largest stability region can be achieved under a desired convergence rate. Then the weighting matrix $M$ is determined by (20) and a feasible initial control law can be determined according to (25).

In the presence of disturbances, the MADS can be estimated by (28). If the given range of disturbances is within the MADS, then the FW-MPC algorithm is feasible and the UUBR can be estimated by (29). Otherwise, we can increase $e$ and then repeat above process until a feasible solution is found.

For the case with system uncertainties, besides conditions (15) and (16), condition (38) must be satisfied when solving the optimization problem (42) offline. This is intended to maximize the stability region under a given range of uncertainties. If there is 
no solution, we can increase $e$ and then repeat until a feasible FW-MPC algorithm is found.

As shown in Lee and Kouvaritakis (1999) and Chen et al (2001), the stability region can be significantly enlarged by introducing $\mu$. However, it should be chosen according to the trade-off between the size of the stability region and the achievable performance, which is defined by the integral part in the performance index (Chen et al, 2001).

\section{Simulation results}

Consider a second order system

$$
\left\{\begin{array}{l}
x_{1}(k+1)=0.875 x_{1}(k)+1.125 x_{2}(k)+u(k) \\
x_{2}(k+1)=0.375 x_{1}(k)+1.625 x_{2}(k)
\end{array}\right.
$$

with control constraint $|u| \leq 1$. It is an unstable plant with one pole outside the unit circle. The prediction horizon is chosen as $N=3$ and the weighting matrices in the performance index (5) are chosen as

$$
Q=\left[\begin{array}{cc}
10 & 0 \\
0 & 10
\end{array}\right], \quad R=1
$$

\subsection{The influence of $e$}

Firstly, influence of $e$ on stability and robustness properties of the new MPC proposed in this paper is investigated. The results are listed in Tab.1, where the volume of MADS is calculated when $C$ in (26) is given by

$$
C=\left[\begin{array}{ll}
1 & 0 \\
0 & 1
\end{array}\right] \text {. }
$$

The second row in Tab.1 shows that the stability region is getting smaller, as $e$ decreases. $e$ implies the convergence rate of the system state under the MPC algorithm. This is also illustrated in Figure 1 in a more intuitive way. A large $e$ implies that the state rapidly contracts to the origion. As shown in Fig.1, the volume of stability region reaches its maximum 11.0207 when $e=1.0001$. When $e$ increases to a critical value, there will be no stability region for the system. This is easy to understand: in this critical case, even 
the extreme control signal (due to the control constraints) cannot push the first predicted state into the sub-region determined by $x^{T}$ eZex $\leq \mu$.

The third row in Tab.1 shows that the maximum allowable system uncertainty increases with $e$. However, when $e$ reaches certain value, e.g., $e=1.8$ for this system, the maximum allowable system uncertainty does not increase significantly any more.

The fourth row in Tab 1 illustrates the influence of $e$ on MADS. When $e$ is small, e.g., $e=1.2$, although the stability region is relatively large, the corresponding MADS is small. A small $e$ implies the small ability to accommodate the influence of the external disturbance or uncertainties. In general, the MADS increases with e. However, as indicated in (28), the MADS depends not only on $e$ but also on $Z$, and, as shown in Figure 1, the stability region which is determined by matrix $Z$ decreases with $e$. This causes that when $e$ is larger than 1.8, increasing e actually reduces the allowable maximum disturbance.

The last row in Tab 1 is to demonstrate the influence of $e$ on the disturbance attenuation level of the FW-MPC algorithm. As shown in Tab 1, at the beginning, the UUBR reduces with e, which implies that the MPC algorithm can attenuate the persistent disturbance to a smaller region. However, since, similar to the MADS, both $e$ and $Z$ have the influence on UUBR as shown in (29), after $e$ is larger than certain value, e.g., $e=1.8$ in this example, the UUBR does not reduce and might even increase.

\subsection{Online control performance}

This simulation is to compare the performance of FW-MPC algorithm proposed in this paper and TW-MPC algorithms. The FW-MPC algorithm is designed with $e=1.8$ while the TW-MPC algorithm is designed with the LMI-base method proposed by Lee (1998), and the terminal weighting matrix for the TW-MPC is determined as

$$
P=\left[\begin{array}{cc}
32.2483 & 91.3477 \\
91.3477 & 390.9721
\end{array}\right]
$$

In order to fairly compare the performances of these MPC algorithms, the summing up cost, i.e., $\sum_{i=0}^{k}\left(x(i)^{T} Q x(i)+u(i)^{T} R u(i)\right), k=0, \ldots, \infty$, is introduced. 
Fig. 2 illustrates the achieved performances of two MPC algorithms in the nominal case, i.e., without any system uncertainty or disturbance. Similar performances are achieved by FW-MPC and TW-MPC.

Fig. 3 and Fig. 4 show the simulation results for the system with uncertainties. In general, the FW-MPC yields better performance under perturbation of the system matrix. In some cases, the system under the TW-MPC becomes unstable while the FW-MPC still exhibits promising performance, as shown in Fig. 4.

The performance of FW-MPC and TW-MPC under bounded disturbances is compared in Fig. 5. When the bounded disturbances with time history shown in the third plot of Fig. 5 are applied to the system, the system under the TW-MPC becomes unstable but FW-MPC still stabilizes the system and attenuates the influence of the disturbance to a certain range, i.e., UUBR. Fig. 6 illustrates that (29) gives a very satisfactory estimate of the UUBR. It is clear in Fig. 6 that the FW-MPC steers the state into the estimated UUBR and then keeps the state within it thereafter under the bounded disturbnaces.

\section{Conclusion}

This paper presents a novel MPC algorithm with promising robustness and disturbance attenuation ability. Different from the most conventional MPC algorithms, where a terminal state weighting is added in the performance index to guarantee stability, the new algorithm employs an additional first predicted state weighting term in the performance index. This provides an alternative way to design stability guaranteed MPC algorithms. As shown by theoretic analysis and numerical simulation, when there are no uncertainties and disturbances, similar stability and performance are achieved by this new algorithm and terminal weighting based MPC algorithms. In the presence of disturbance and uncertainty, the new MPC algorithm exhibits much better disturbance attenuation ability and robustness.

\section{Acknowledgements}

This work is supported by an ORS Award from the Overseas Research Students Awards Scheme, Universities UK. 
References

BEMPORAD, A. 1998: A predictive controller with artificial Lyapunov function for linear systems with input/state constraints. Automatica, 34(10), 1255-1260.

BITMEAD, R.R., GEVERS, M. and WERTZ, V. 1990: Adaptive Optimal Control: The Thinking Man's GPC. Trentice-Hall, New York.

CHEN, H. and ALLGOWER, F. 1998: A quasi-infinite horizon nonlinear model predictive control scheme with guaranteed stability. Automatica, 34(10), 335-348.

CHEN, W.-H., BALANCE, D.J. and O'REILLY, J. 2000: Model predictive control of nonlinear systems: Computational burden and stability. IEE Proc.-Control Theory Appl., 147(4), 387-394.

CHEN, W.-H., BALANCE, D.J. and O'REILLY, J. 2001: On attraction domain of model predictive control of nonlinear systems with input/state constraints. Proceedings of American Control Conference, Chicago, Illinois, USA.

CLARKE, D.W. 1994: Advances in Model-based Predictive Control. Oxford University Press.

CORLESS, M. J. and LEITMANN, G. 1981: Continuous state feedback guaranteeing uniform ultimate boundedness for uncertain dynamic systems. IEEE Trans. Autom. Control, AC-26(5), 1139-1144.

GARCIA, C.E., PRETT, D.M. and MORARI, M. 1989: Model Predictive Control: Theory and Practice - a Survey. Automatica, 25(3), 335-348.

KOTHARE, M.V., BALAKRISHNAN, V. and MORARI, M. 1996: Robust constrained model predictive control using linear matrix inequalities. Automatica, 32(10), pp. 1361-1379.

KOTHARE, S. L. O. and MORARI, M. 2000: Contractive model predictive control for constrained nonlinear systems. IEEE Trans. Autom. Control, 45(6), 1053-1071.

KOUVARITAKIS, B., ROSSITER, J. A. and SCHUURMANS, J. 2000: Efficient robust predictive control. IEEE Trans. Autom. Control, 45(6), 1545-1549.

KOUVARITAKIS, B., CANNON, M., and ROSSITER, J. A. 2002: Who needs QP for linear MPC anyway? Automatica, 38, 879-884.

LEE, J. W., KWON, W. H. and CHOI, J. 1998: On stability of constrained receding horizon control with finite terminal weighting matrix. Automatica, 34(12), 1607-1612. 
LEE, Y. I. and KOUVARITAKIS, B. 1999: Stabilizable regions of receding horizon predictive control with input constraints. System \& Control Letters, 38, 13-20.

MACIEJOWSKI, J.M. 2002: Predictive Control with Constraints. Personal Education Limited, Harlow, Essex, UK.

MAYNE, D.Q., RAWLINGS, J.B., RAO, C.V. and SCOKAERT, P.O.M. 2000: Survey Paper-Constrained model predictive control: Stability and optimality. Automatica, 36, 789-814.

MAYNE, D.Q. and MICHALSKA, H. 1990: Receding horizon control of nonlinear systems, IEEE Trans. Autom. Control, 35(7), 814-824.

MICHALSKA, H. and MAYNE, D.Q. 1993: Robust receding horizon constrained nonlinear systems. IEEE Trans. Autom. Control, 38(11), 1623-1633.

POLAK, E. and YANG, T.H. 1993: Moving horizon control of linear systems with input saturation and plant uncertainty: part 1. Robustness. INT. J. Control, 58(3), 613-638.

POLAK, E. and YANG, T.H. 1993: Moving horizon control of linear systems with input saturation and plant uncertainty: part 2. Disturbance rejection and tracking. INT. J. Control, 58(3), 639-663.

RAWLINGS, J.B. and MUSKE, K.R. 1993: The stability of constrained receding horizon control. IEEE Trans. Autom. Control, 38(10), 1512-1516.

SCOKAERT, P.O.M., RAWLINGS, J.B. and MEADOWS, E.S. 1997: Discrete-time stability with perturbations: Application to Model Predictive Control. Automatica, 33(3), 463-470.

SZNAIER, M. and DAMBORG, M.J. 1990: Heuristically enhanced feedback control of constrained discrete-time linear systems. Automatica, 26(3), 521-532. 
Table 1 The influence of $e$

\begin{tabular}{|c|c|c|c|c|c|c|}
\hline$e$ & 1.2 & 1.4 & 1.6 & 1.8 & 2.0 & 2.2 \\
\hline Volume of stability region & 8.7660 & 7.1082 & 5.6601 & 4.3145 & 3.1889 & 2.3289 \\
\hline Maximum allowable $A_{u}$ & $\pm 8 \% A_{0}$ & $\pm 14 \% A_{0}$ & $\pm 18 \% A_{0}$ & $\pm 22 \% A_{0}$ & $\pm 24 \% A_{0}$ & $\pm 25 \% A_{0}$ \\
\hline Volume of MADS & 0.2435 & 0.5803 & 0.7960 & 0.8522 & 0.7958 & 0.6929 \\
\hline Volume of UUBR & 8.7660 & 2.9422 & 1.8279 & 1.4848 & 1.4505 & 1.5684 \\
\hline
\end{tabular}

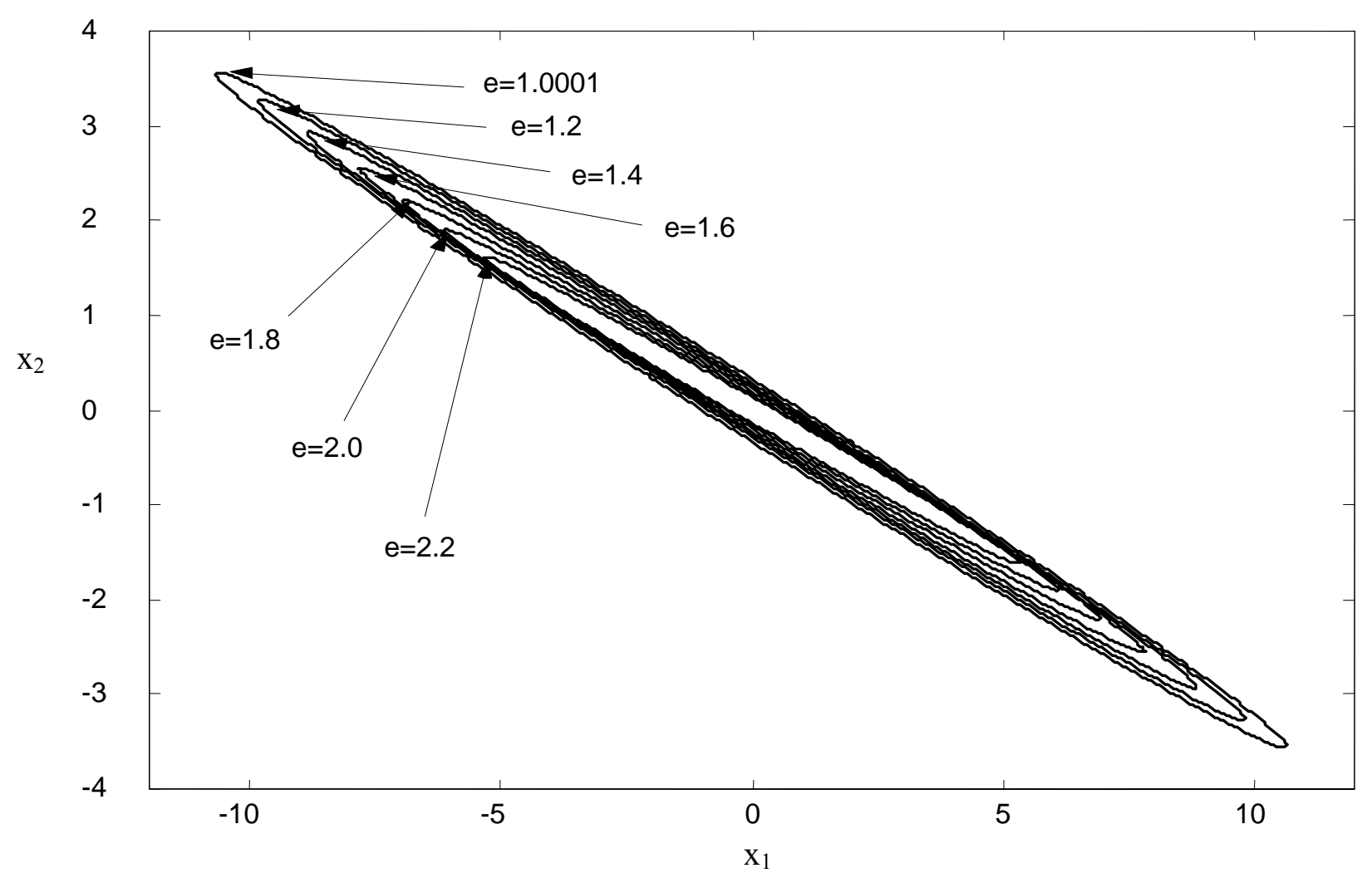

Fig. 1. Stability regions 

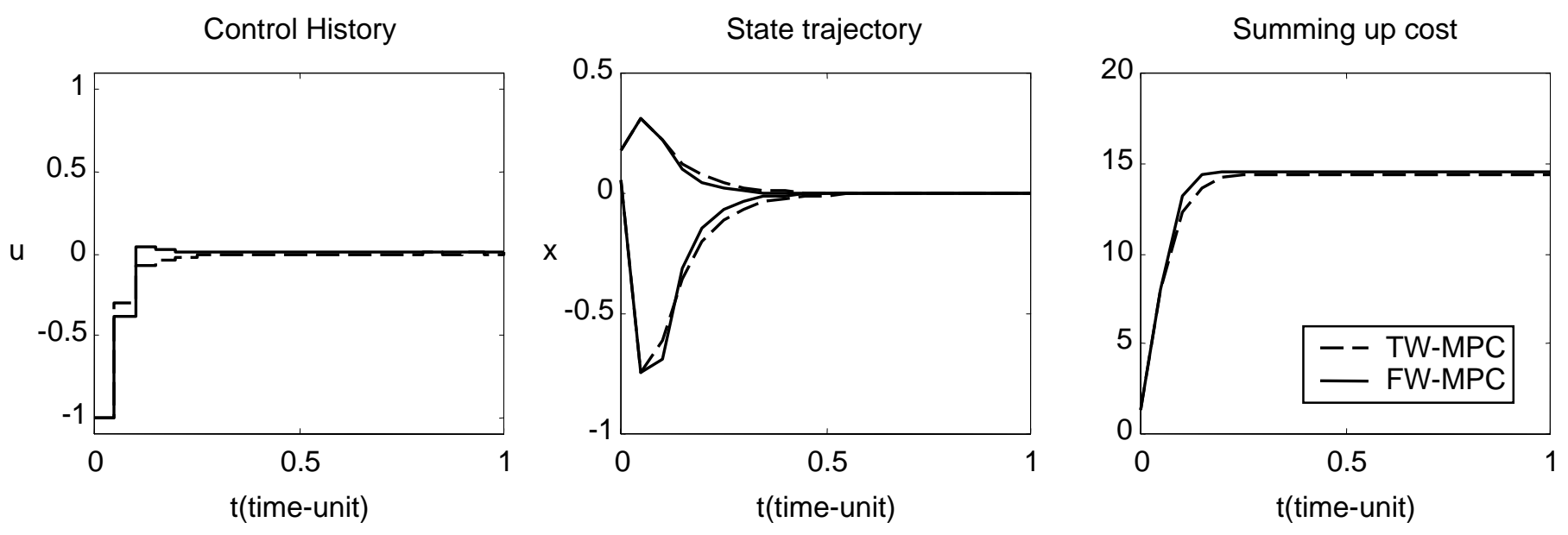

Fig. 2. Online control performance: no system uncertainties or disturbances
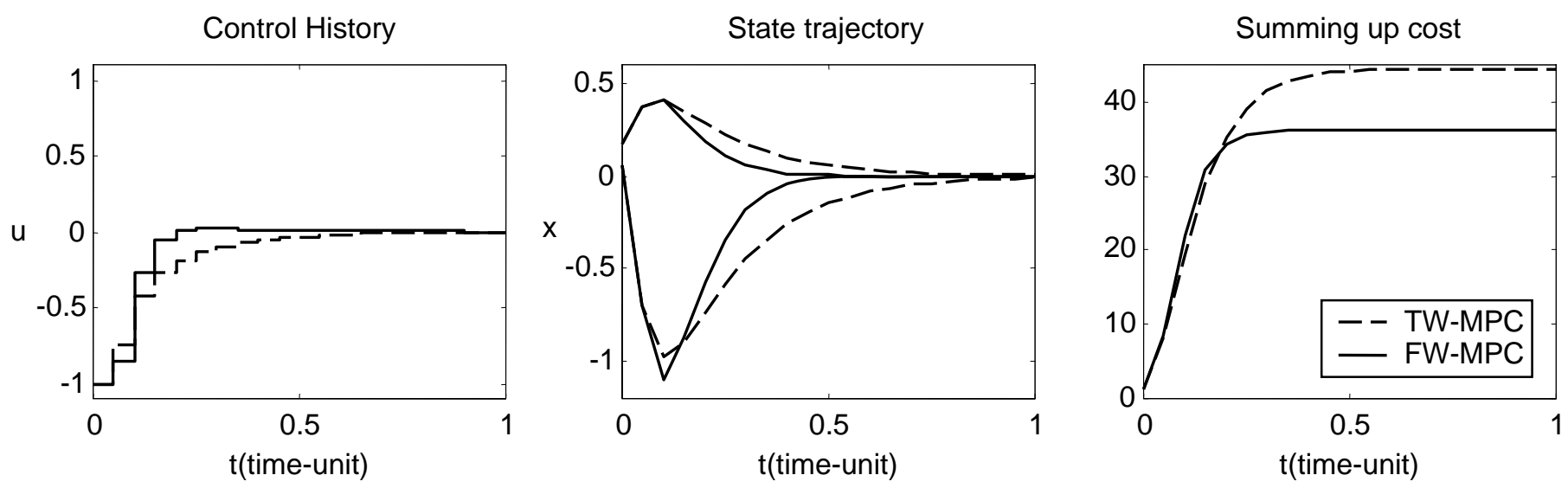

Fig. 3. Online control performance: with system uncertainty $A_{u}=0.2 A_{0}$
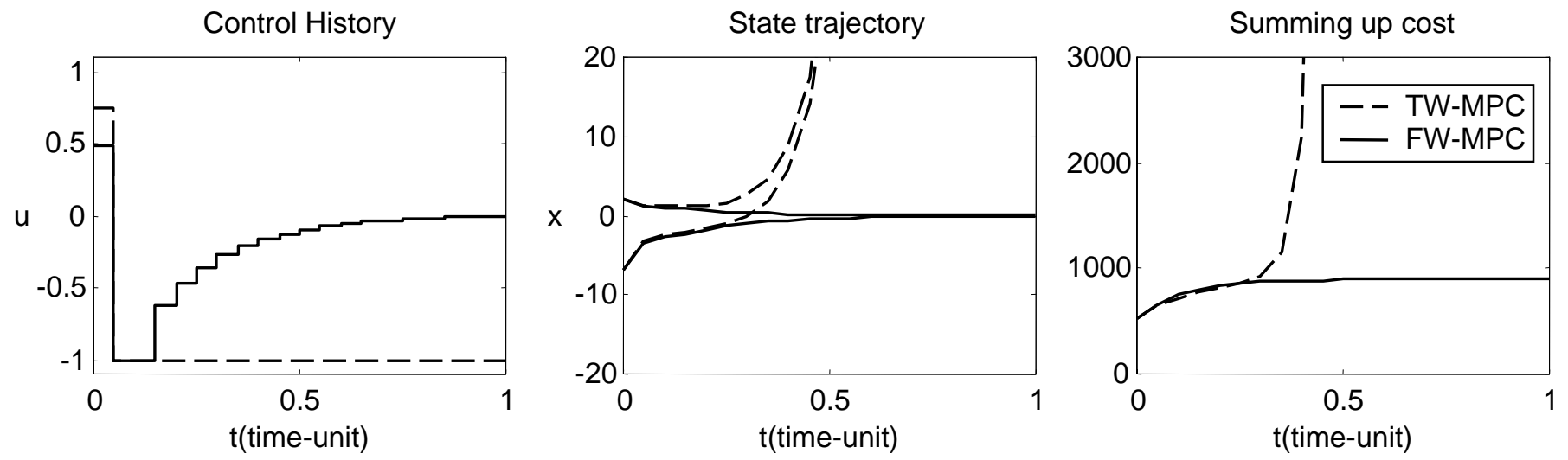

Fig. 4. Online control performance: with system uncertainty $A_{u}=\left[\begin{array}{llll}0.05 & 0 ; & 0 & 0.15\end{array}\right]$ 

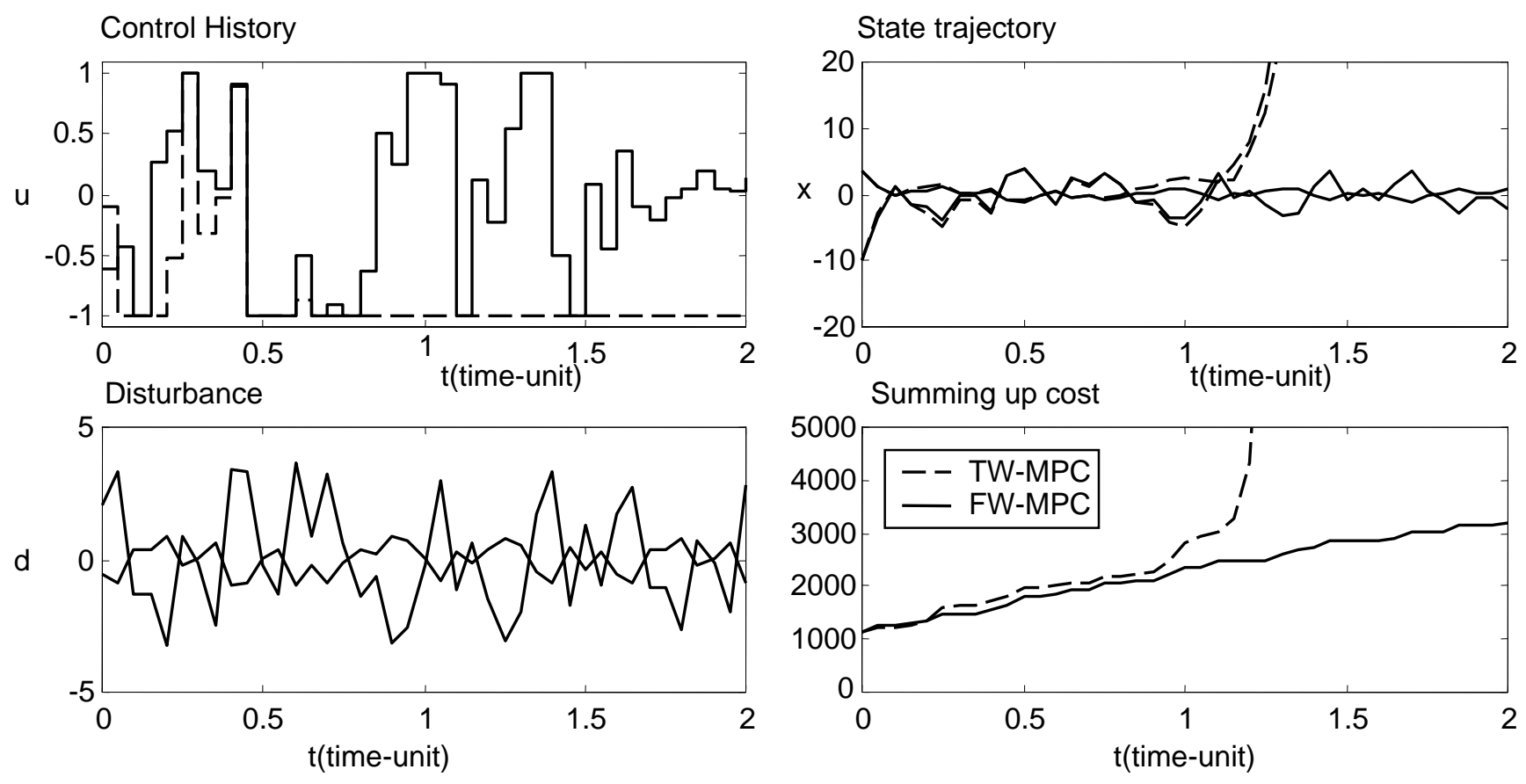

Fig. 5. Online control performance under bounded disturbances

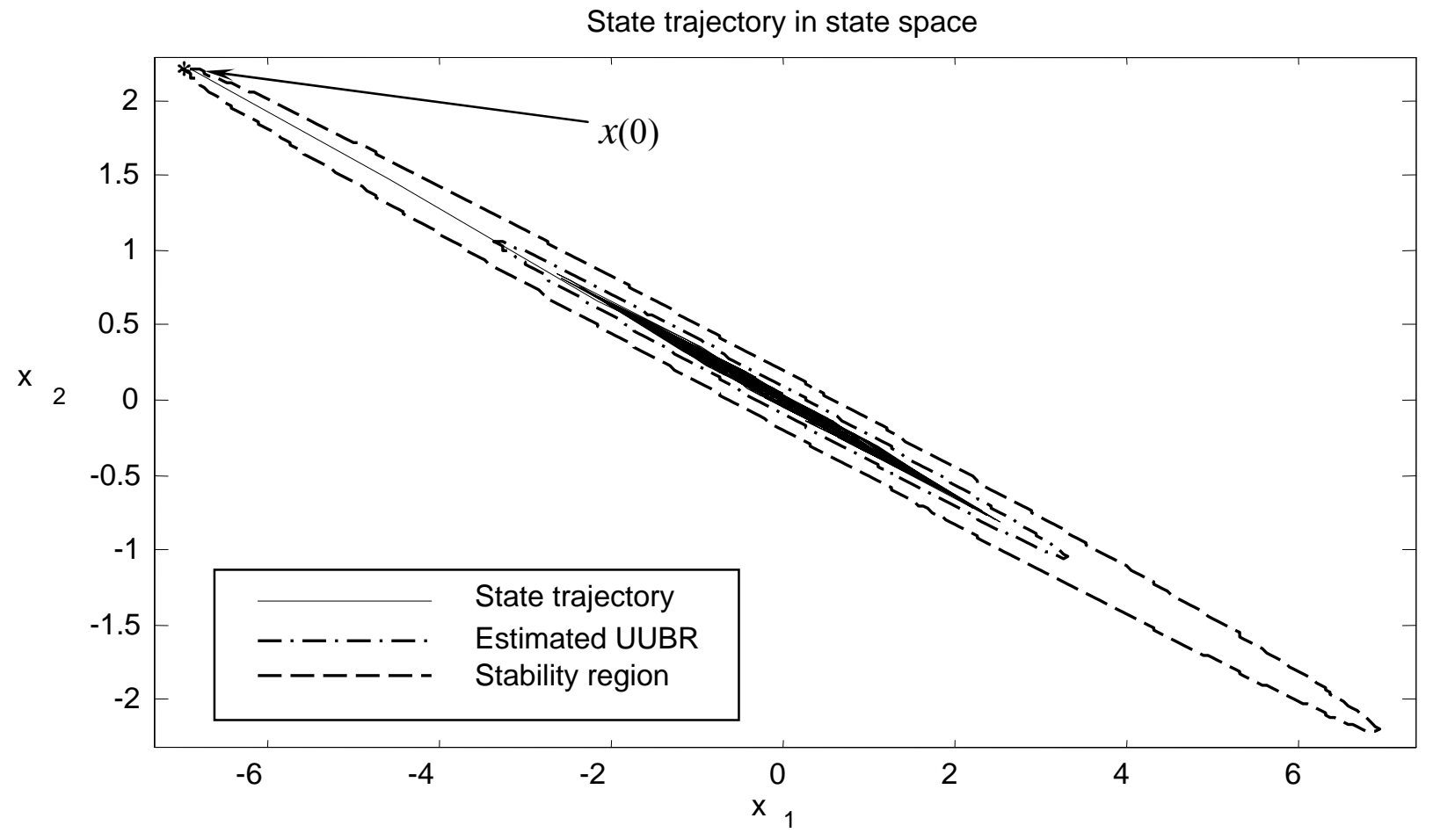

Fig. 6. Stability region ( $e=1.8)$, estimated UUBR and state trajectory 